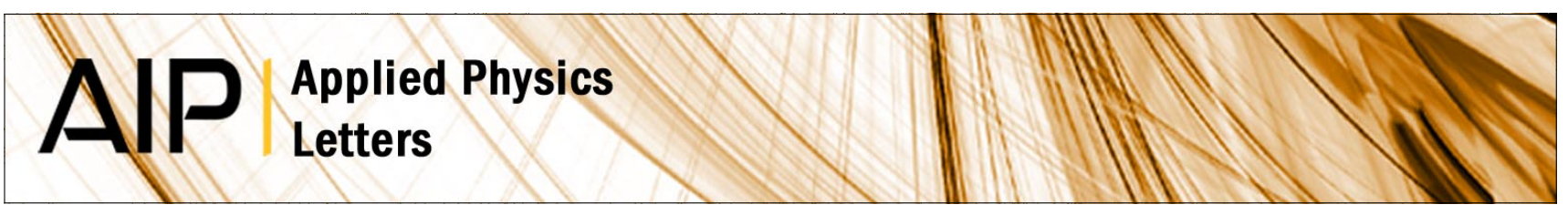

\title{
Hole recapture limited single photon generation from a single n-type charge-tunable quantum dot
}

P. A. Dalgarno, J. McFarlane, D. Brunner, R. W. Lambert, B. D. Gerardot et al.

Citation: Appl. Phys. Lett. 92, 193103 (2008); doi: 10.1063/1.2924315

View online: http://dx.doi.org/10.1063/1.2924315

View Table of Contents: http://apl.aip.org/resource/1/APPLAB/v92/i19

Published by the American Institute of Physics.

\section{Related Articles}

Spin accumulation in parallel-coupled quantum dots driven by a symmetric dipolar spin battery J. Appl. Phys. 111, 053708 (2012)

Enhancement of thermoelectric efficiency and violation of the Wiedemann-Franz law due to Fano effect J. Appl. Phys. 111, 053704 (2012)

Tunable spin manipulation in a quantum dot embedded in an Aharonov-Bohm interferometer J. Appl. Phys. 111, 054306 (2012)

Back-action-induced non-equilibrium effect in electron charge counting statistics Appl. Phys. Lett. 100, 092112 (2012)

Modulating quantum transport by transient chaos

Appl. Phys. Lett. 100, 093105 (2012)

\section{Additional information on Appl. Phys. Lett.}

Journal Homepage: http://apl.aip.org/

Journal Information: http://apl.aip.org/about/about_the_journal

Top downloads: http://apl.aip.org/features/most_downloaded

Information for Authors: http://apl.aip.org/authors

\section{ADVERTISEMENT}

\section{NEW!}

iPeerReview

AlP's Newest App

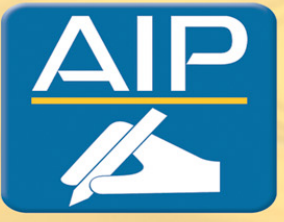

Authors...

Reviewers...

Check the status of

submitted papers remotely!

ADPublishing 


\title{
Hole recapture limited single photon generation from a single $n$-type charge-tunable quantum dot
}

\author{
P. A. Dalgarno, ${ }^{1, a)}$ J. McFarlane, ${ }^{1}$ D. Brunner, ${ }^{1}$ R. W. Lambert, ${ }^{1}$ B. D. Gerardot,${ }^{1}$ \\ R. J. Warburton, ${ }^{1}$ K. Karrai, ${ }^{2}$ A. Badolato, ${ }^{3}$ and P. M. Petroff ${ }^{3}$ \\ ${ }^{1}$ School of Engineering and Physical Sciences, Heriot-Watt University, Edinburgh EH14 4AS, \\ United Kingdom \\ ${ }^{2}$ Center for NanoScience, Department für Physik der LMU, Geschwister-Scholl-Platz 1, \\ 80539 Munich, Germany \\ ${ }^{3}$ Materials Department, University of California, Santa Barbara, California 93106, USA
}

(Received 12 February 2008; accepted 19 April 2008; published online 13 May 2008)

\begin{abstract}
The complete control of the electron occupation of a single InGaAs dot is shown to produce highly antibunched single photon emission with nonresonant optical excitation. Intensity correlation measurements show $g^{(2)}(0)$ values of $3 \%(50 \%)$ at low (high) excitation power. A distinct double peak structure is shown at time zero, demonstrating that although two photons may be emitted per excitation pulse, they are not simultaneously emitted. We interpret this feature as a hole recapture process from the wetting layer into the dot after initial recombination. The recapture dynamics is shown to be adjustable through engineering the valence potential. (C) 2008 American Institute of Physics. [DOI: 10.1063/1.2924315]
\end{abstract}

A single self-assembled semiconductor quantum dot (SAQD) is a promising candidate for a practical single photon source (SPS). The primary prerequisite of a SPS, photon antibunching, has been demonstrated in various quantum dot systems. ${ }^{1,2}$ Unlike single molecules, SAQDs do not experience photobleaching or photoblinking, ${ }^{3}$ unlike deep level nitrogen vacancies in diamond they have no shelving states, ${ }^{4}$ and they are more practical for long term use than trapped single atoms. ${ }^{5}$ In addition, SAQDs can emit over a wide spectral range depending on material choices, including $1.3 \mu \mathrm{m}{ }^{6}$ Polarization entangled photon pairs, a useful resource for quantum information processing, have been produced in the biexciton decay from single SAQDs. ${ }^{7,8}$ A drawback in using SAQDs is photon extraction efficiencies from the high refractive index semiconductor. However, SAQD have been successfully incorporated into microcavity systems and collection efficiencies of $\sim 40 \%$ have been recently reported. ${ }^{9}$

Photon antibunching is quantified through a measurement of the second order intensity correlation at time zero $g^{(2)}(0)$. An ideal single photon source would have a $g^{(2)}(0)$ of zero. For quantum dot systems, resonant excitation is a promising route to achieving an ultralow $g^{(2)}(0)$. Background emission from the wetting layer, other dots or sample defects are avoided. In addition, as excitonic excitations in the ground state of a QD resembles a true two level system, a $\pi$ pulse results in a complete population inversion. ${ }^{10}$ However, with resonant excitation there are significant challenges in distinguishing the PL from the excitation light. ${ }^{10}$ On the other hand, nonresonant excitation offers no such difficulty and is compatible with electrical injection using $p-i-n$ diode devices. ${ }^{11}$ However, with nonresonant excitation a low $g^{(2)}(0)$ can be difficult to achieve due to background emission. Furthermore, flooding the dot with carriers potentially pollutes the SPS emission with timing jitter. In this letter, we use a charge-tunable structure to take precise control of the majority carrier (electrons) in a single dot to produce highly

\footnotetext{
${ }^{a)}$ Electronic mail: p.a.dalgarno@hw.ac.uk.
}

antibunched single photon emission from a known exciton configuration with nonresonant excitation. We subsequently demonstrate that hole recapture from the wetting layer is the limiting factor in achieving a zero $g^{(2)}(0)$.

Charge-tunable SAQD devices allow for precise control over exciton charge via Coulomb blockade. ${ }^{12}$ In our device a $n$-type back contact is separated from annealed InGaAs/GaAs dots by a $25 \mathrm{~nm}$ GaAs barrier. A capping layer of either $30 \mathrm{~nm}$ (sample A) or $10 \mathrm{~nm}$ (sample B) separates the dots from an AlAs/GaAs blocking barrier (Fig. 1). A dc bias between a NiCr Schottky gate on the device surface and the back contact allows for controlled tunneling of electrons into the dots on time scales of $\sim 10$ ps. ${ }^{12}$

PL is detected using a low temperature microscope at $5 \mathrm{~K}$. Excitation is provided by a nonresonant, $1.50 \mathrm{eV}$ $(826 \mathrm{~nm})$, pulsed laser at $20 \mathrm{MHz}$ with a measured full width at half maximum of $\sim 50 \mathrm{ps}$. Excitation power is adjusted through the use of optical attenuators in order to preserve the temporal properties of the laser. The dots emit at around $950 \mathrm{~nm}$. Refractive index mismatch between the sample and surrounding medium (He exchange gas) is reduced through the use of a cubic zirconium $(n=2.15)$ supersolid immersion lens ( $s$-SIL). The $s$-SIL increases the collection efficiency from $\sim 0.8 \%$ to $\sim 8 \%$ and is easier to implement than a microcavity. In addition, the $s$-SIL decreases the diffraction limited spot size to $270 \mathrm{~nm}$, improving spatial resolution. Correlation measurements are per-
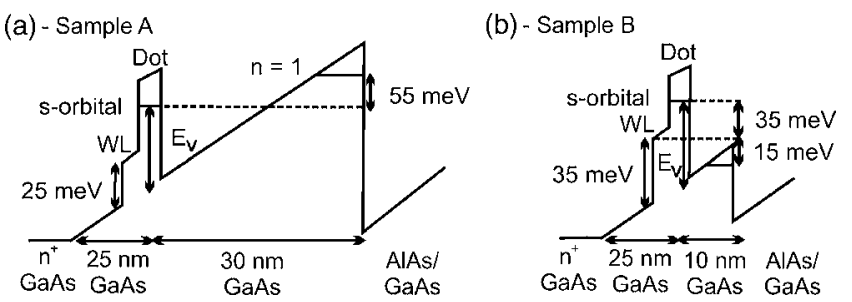

FIG. 1. Schematic of the valence band profile of samples (a) A and (b) B (not to scale). In (a), the 2D well valence states formed at the capping layer blocking barrier interface have the highest energy. In (b) the WL is energetically lower than the interface states. $E_{v}$ is approximately $75 \mathrm{meV}$ for both samples. All energies are accurate to around $\pm 10 \mathrm{meV}$. 

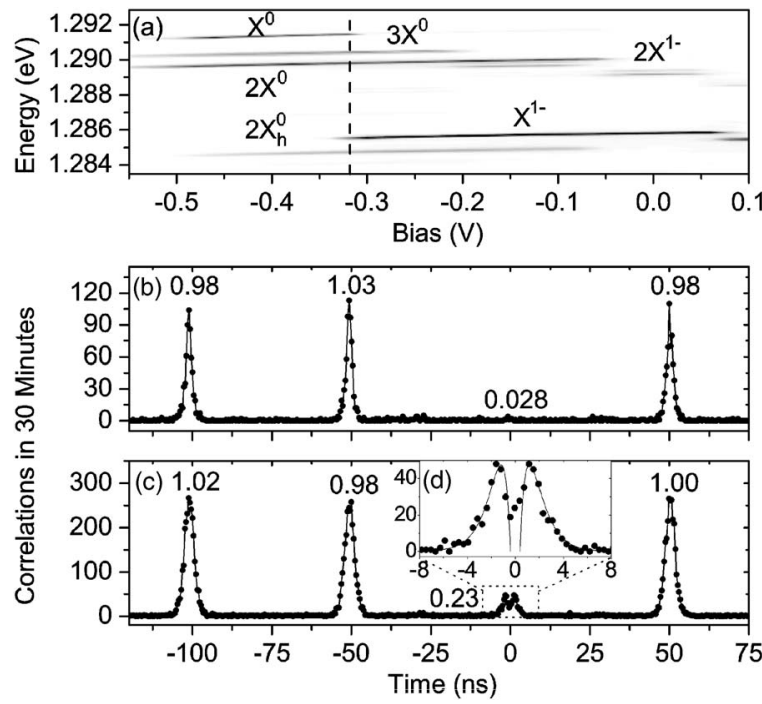

FIG. 2. (a) Time-integrated PL from a single dot (sample A) at $5 \mathrm{~K}$ with excitation power $P_{\text {sat }}=2 \mu \mathrm{W} / \mu \mathrm{m}^{2}$. White (black) corresponds to 120 (2000) counts. The neutral exciton $X^{0}$ and negatively charged exciton $X^{1-}$ are labeled, along with biexciton $\left(2 X^{0}\right)$ and triexciton $\left(3 X^{0}\right)$ related features. (b) and (c) show example intensity autocorrelation data from $X^{1-}$ taken at similar biases but at different excitation powers, $0.02 P_{\text {sat }}$ and $P_{\text {sat }}$, respectively. The values above the peaks are the peak areas after normalization to the nontime zero peaks. (d) shows a magnified view of the time zero feature from (c). The solid line is a fit of the rise and fall times of the two peaks using $\tau_{\text {rise }}=0.6 \mathrm{~ns}$ and $\tau_{\text {fall }}=1.05 \mathrm{~ns}$.

formed using a custom built Hanbury Brown-Twiss (HBT) spectrometer. The HBT uses a high efficiency (over 90\%) polarization insensitive transmission grating to spectrally filter the PL with a resolution of $350 \mu \mathrm{eV}$. This is key as the HBT allows for the precise selection of individual exciton lines without contamination from other exciton emission. Correlation data is taken using two silicon single photon avalanche diodes (SPADs) and time correlated single photon counting electronics. Each SPAD has a timing jitter of approximately $400 \mathrm{ps}$. The temporal response of the system is therefore entirely limited by the timing jitter of the SPADs.

Figure 2(a) shows the PL from a single dot from sample $\mathrm{A}$ as a function of bias. The change in Coulomb interactions with exciton charge brings about discrete steps in emission energy. The neutral exciton $X^{0}$ and negatively charged exciton $X^{1-}$ are labeled. As excitation power increases, the PL intensities of both $X^{0}$ and $X^{1-}$ linearly increases until a maximum intensity is reached at the saturation power $P_{\text {sat }}$. As excitation power is increased beyond $P_{\text {sat }}$ the PL intensity decreases. For the dot shown in Fig. $1 P_{\text {sat }}$ is $\sim 2 \mu \mathrm{W} / \mu \mathrm{m}^{2}$. Figure 2(b) shows intensity correlation data from the $X^{1-}$ emission at the center of the voltage plateau at an excitation power $0.05 P_{\text {sat }}$, where the biexciton PL is approximately half the intensity of the $X^{0} \mathrm{PL}$. At this power, the photon flux is reasonable, 3000 counts/s per SPAD, giving excellent signal to noise in our $g^{(2)}(0)$ measurements. $g^{(2)}(0)$ is quantified by determining the area of the time zero feature relative to the area of the nontime zero peaks. We determine $g^{(2)}(0)$ to be $\sim 0.03$, corresponding to a $3 \%$ probability that two or more photons are emitted per excitation pulse. We see neither a background floor between the side peaks nor long-lived memory effects, which manifest themselves through the reduction of the side peaks near to time zero. ${ }^{2}$ As excitation power is increased, we record a significant increase in $g^{(2)}(0)$. At $P_{\text {sat }}$, the SPAD counts double and $g^{(2)}(0)$ is 0.23 [Fig. 2(a)]. For comparison, $g^{(2)}(0)$ values between 0.04 and

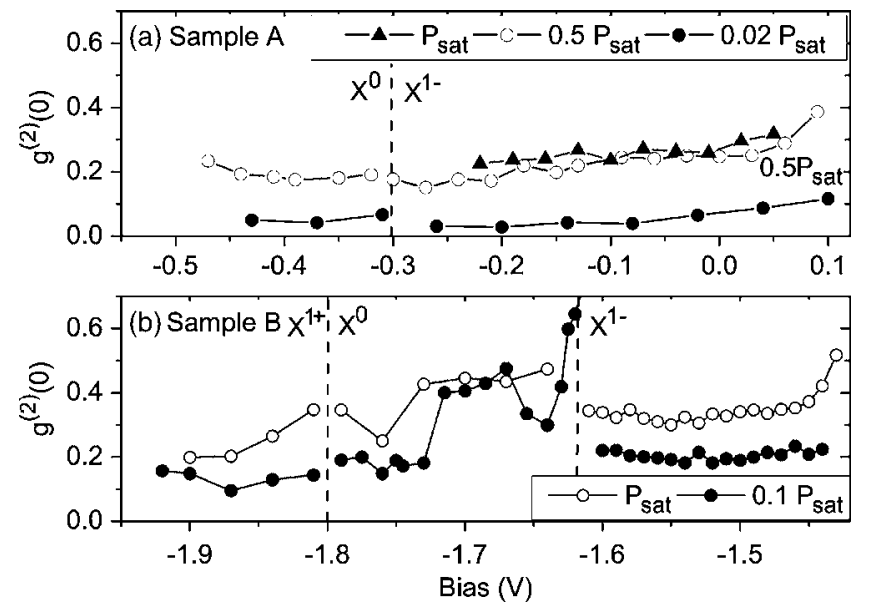

FIG. 3. The measured $g^{(2)}(0)$ values as a function of bias and excitation power for (a) $X^{0}$ and $X^{1-}$ from the dot shown in Figs. 2(a) and (b) for $X^{0}$, $X^{1-}$, and $X^{1+}$ from a dot from sample $B . P_{\text {sat }}$ is $\sim 2.0 \mu \mathrm{W} / \mu \mathrm{m}^{2}$ for sample A and $2.0 \mu \mathrm{W} / \mu \mathrm{m}^{2}$ for sample B. At equivalent excitation density $g^{(2)}(0)$ is larger, by approximately a factor of 2 , for sample A than for sample B.

0.4 have been reported for similar dots in non-charge-tunable structures with both electrical injection and nonresonant optical excitation., ${ }^{2,911}$ Figure 3(a) shows $g^{(2)}(0)$ values for $X^{0}$ and $X^{1-}$ at three excitation powers as a function of bias. There is a bias dependence of $g^{(2)}(0)$ for both excitons. $g^{(2)}(0)$ does not change with exciton charge. Measurements on a further 5-6 dots from the same sample show a fluctuation of $g^{(2)}(0)$ of around $25 \%$ from dot to dot at equivalent excitation power.

Our data allow us to determine the origins of the nonzero $g^{(2)}(0)$. Contributions to the PL from background emission is negligible as no such emission is seen in the PL spectra within the full 16-bit dynamic range of our CCD camera. Contamination from other dots or excitons can be ruled out as at any particular bias the energy separations are larger than the detection bandwidth [Fig. 2(a)]. Figure 2(d) highlights the structure of the $g^{(2)}(0)$ feature in Fig. 2(b). At time zero there is a clear minimum in correlations. However, within a few nanoseconds, there is an increase in correlations. This implies there is a carrier recapture process within the dot leading to a reforming of an exciton and subsequent reemission of a photon within the excitation cycle. ${ }^{13}$ The charge-tunable device provides complete control over the electron dynamics. In the plateau center the ground state exciton is established by electron tunneling. ${ }^{12}$ It can therefore be concluded that the recapture process is related to hole dynamics. The recapture dynamics considerably vary from dot to dot. On average only one third of the dots studied from sample A show a clear splitting of the $g^{(2)}(0)$ feature.

We have previously reported that under nonresonant excitation a positive space charge region is formed in the device at the interface between the capping layer and the blocking barrier. ${ }^{14}$ The interface can be modeled as a twodimensional (2D) triangular well and the energy of the valence $s$ orbital in the dot determined with a phenomological Coulomb blockade model. ${ }^{14}$ The hole density at this interface is estimated from the voltage shift of charging events with excitation power to be under 1000 holes $/ \mu \mathrm{m}^{2}$ at $P_{\text {sat }}$. Therefore, only the lowest energy level $n=1$ in the $2 \mathrm{D}$ well is occupied by holes at $5 \mathrm{~K}$. At the low bias end of the $X^{1-}$ plateau, $\sim-0.3 \mathrm{~V}$, the $n=1$ level is approximately $55 \pm 10 \mathrm{meV}$ above the valence $s$ orbital in the dot. This 


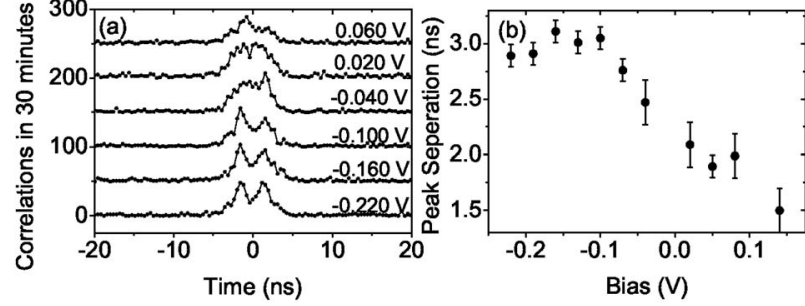

FIG. 4. (a) Evolution of the central $g^{(2)}(0)$ peak from $X^{1-}$ as a function of bias for sample A. Each curve is offset by 50 correlations from the previous curve. (b) Measured peak to peak seperation of the $g^{(2)}(0)$ feature from (a) as a function of bias. (a) contains a subset of the data used for (b).

decreases to around $10 \mathrm{meV}$ at the high bias end of the $X^{1-}$ plateau. As holes trapped in the interface will diffuse laterally away from the dot, there is close to zero probability of dot repopulation via phonon-assisted carrier recapture of holes from the interface states at $5 \mathrm{~K}$. However, excitation is above the wetting layer (WL) bandgap, $1.46 \mathrm{eV}$. Using the $1.52 \mathrm{eV}$ bandgap of GaAs and a WL conduction band to valence band confinement energy offset ratio of 0.58 (Ref. 15) the WL valence states are estimated to be $50 \mathrm{meV}$ below the valence $s$ orbital in the dot. There is a probability that some holes are trapped in localized potential fluctuations in the WL. ${ }^{16}$ These trapped holes are then free to repopulate the dot after recombination. Once a hole is present in the dot, the dot will automatically capture either a single electron $X^{0}$ or two electrons $X^{1-}$, depending on bias. The reformation of an exciton gives rise to secondary photon emission. The varying nature of the WL potential across each sample is reflected in the varying recapture dynamics seen from dot to dot. An increase in excitation power increases the WL hole density and increases the recapture probability. It is expected that the $g^{(2)}(0)$ feature at $0.05 P_{\text {sat }}$ shows a similar splitting to the higher power data, only it is unresolvable due to the reduced correlations.

We estimate the recapture and reemission times, $\tau_{\text {cap }}$ and $\tau_{\text {rad }}$, respectively, in the limit of large time zero splitting by fitting the rise and fall times of the correlations nearest to time zero in Fig. 2(d) to a simple three level rate equation model,,${ }^{17} g^{2}(t) \propto\left[\exp \left(-t / \tau_{\text {cap }}\right)-\exp \left(-t / \tau_{\text {rad }}\right)\right] /\left(\tau_{\text {cap }}-\tau_{\text {rad }}\right)$. We determine $\tau_{\text {cap }}=0.6 \mathrm{~ns}$ and $\tau_{\text {rad }}=1.05 \mathrm{~ns}$ for the data shown in Fig. 2(d). Accounting for the $400 \mathrm{ps}$ instrumental response $\tau_{\text {rad }}$ is in good agreement with the directly measured lifetime of $X^{1-}$ from the same dot $(0.97 \mathrm{~ns})$. We estimate that the recapture time of an exciton is $\sim 450 \mathrm{ps}$. The hole recapture process highlights an important feature of our sample. Although two or more photons are sometimes emitted per excitation pulse, they are not simultaneously emitted. We are confident that with faster detectors, $<100 \mathrm{ps}, g^{(2)}(0)$ would reach zero.

Our interpretation is consistent with an increasing $g^{(2)}(0)$ with decreasing electric field, Fig. 3(a). As the field decreases hole tunneling from the WL into the interface is reduced and the hole density in the WL increases, increasing the probability of hole recapture. Further evidence for the bias dependent recapture probability is seen in the close up of the time zero feature as a function of bias for $X^{1-}$ [Fig. 4(a)]. As the bias increases the splitting reduces, Fig. 4(b), corresponding to a faster hole recapture time.

We confirm our interpretation through engineering of the valence band to alter the hole recapture probability. For sample B, the capping layer thickness is reduced from 30 to $10 \mathrm{~nm}$ [Fig. 1(b)]. Figure 3(b) shows $g^{(2)}(0)$ as a function of bias for $X^{0}, X^{1-}$, and $X^{1+}$ from a single dot from sample B $\left(X^{0}\right.$ emission at $\left.1.33 \mathrm{eV}\right)$ at $X^{0}$ and $X^{1-}$ saturation powers, $P_{\text {sat }}$, and at $0.1 P_{\text {sat }}$. On average $10 \times$ less excitation power is required to saturate dots from sample $B$ than sample A. Statistics on 6 dots show a $g^{(2)}(0)$ fluctuation of around $25 \%$. Comparing results from both samples at equivalent powers relative to saturation, $g^{(2)}(0)$ is approximately a factor of 2 larger for sample B than sample A. This is reflective of an increased hole recapture probability. The thinner capping layer moves the $n=1$ valence level in the $2 \mathrm{D}$ triangular well to approximately 50(15) meV below the valence $s$ orbital in the dot (WL), Fig. 1(b). Holes are no longer able to relax from the WL into the interface and a greater hole density in the WL is formed. Consequently, the hole recapture probability is increased and $g^{(2)}(0)$ increases. As holes are no longer lost to the interface states less excitation power is required to saturate dots from sample B than sample A. Similar to sample A, only one third of the dots studied from sample B show a clear splitting of the $g^{(2)}(0)$ feature.

In conclusion we have measured a $g^{(2)}(0)$ of 0.03 from a single quantum dot with nonresonant optical excitation. We have demonstrated that the $g^{(2)}(0)$ is limited solely by the hole dynamics in our $n$-type charge-tunable devices. Only with complete control over both electron and hole dynamics will zero $g^{(2)}(0)$ be realized in SAQD with nonresonant excitation. A potential solution may be a double insulated $p-i-n$ heterostructure device and an alternating voltage source. ${ }^{18}$

${ }^{1}$ A. J. Shields, Nat. Photonics 1, 215 (2007).

${ }^{2}$ C. Santori, D. Fattal, J. Vuckovic, G. S. Solomon, and Y. Yamamoto, New J. Phys. 6, 89 (2004).

${ }^{3}$ B. Lounis and W. E. Moerner, Nature (London) 407, 491 (2000).

${ }^{4}$ C. Kurtsiefer, S. Mayer, P. Zarda, and H. Weinfurter, Phys. Rev. Lett. 85, 290 (2000).

${ }^{5}$ B. Darquie, M. P. A. Jones, J. Dingjan, J. Beugnon, S. Bergamini, Y. Sortais, G. Messin, A. Browaeys, and P. Grangier, Science 309, 454 (2005).

${ }^{6}$ P. M. Intallura, M. B. Ward, O. Z. Karimov, Z. L. Yuan, P. See, A. J. Shields, P. Atkinson, and D. A. Ritchie, Appl. Phys. Lett. 91, 161103 (2007).

${ }^{7}$ R. M. Stevenson, R. J. Young, P. Atkinson, K. Cooper, D. A. Ritchie, and A. J. Shields, Nature (London) 439, 179 (2006).

${ }^{8}$ N. Akopian, N. H. Lindner, E. Poem, Y. Berlatzky, J. Avron, D. Gershoni, B. D. Gerardot, and P. M. Petroff, Phys. Rev. Lett. 96, 130501 (2006).

${ }^{9}$ S. Strauf, N. G. Stoltz, M. T. Rakher, L. A. Coldren, P. M. Petroff, and D. Bouwmeester, Nat. Photonics 1, 704 (2007).

${ }^{10}$ A. Muller, E. B. Flagg, P. Bianucci, X. Y. Wang, D. G. Deppe, W. Ma, J. Zhang, G. J. Salamo, M. Xiao, and C. K. Shih, Phys. Rev. Lett. 99, 187402 (2007)

${ }^{11}$ Z. L. Yuan, B. E. Kardynal, R. M. Stevenson, A. J. Shields, C. J. Lobo, K. Cooper, N. S. Beattie, D. A. Ritchie, and M. Pepper, Science 295, 102 (2002).

${ }^{12}$ J. M. Smith, P. A. Dalgarno, R. J. Warburton, A. O. Govorov, K. Karrai, B. D. Gerardot, and P. M. Petroff, Phys. Rev. Lett. 94, 197402 (2005).

${ }^{13}$ T. Aichele, V. Zwiller, and O. Benson, New J. Phys. 6, 1367 (2004).

${ }^{14}$ S. Seidl, M. Kroner, P. A. Dalgarno, J. M. Smith, A. Hogele, M. Ediger, B. D. Gerardot, J. M. Garcia, P. M. Petroff, K. Karrai, and R. J. Warburton, Phys. Rev. B 72, 195339 (2005).

${ }^{15}$ J. Brubach, A. Y. Silov, J. E. M. Haverkort, W. van der Vleuten, and J. H. Wolter, Phys. Rev. B 59, 10315 (1999).

${ }^{16}$ E. S. Moskalenko, M. Larsson, W. V. Schoenfeld, P. M. Petroff, and P. O. Holtz, Phys. Rev. B 73, 155336 (2006).

${ }^{17}$ G. Bastard, Wave Mechanics Applied to Semiconductor Heterostructures (Halsted, New York, 1988).

${ }^{18}$ A. Imamoglu and Y. Yamamoto, Phys. Rev. Lett. 72, 210 (1994). 